\title{
Possibilities of using the HPLC method in the taxonomy of the genus Nitraria (Nitrariaceae)
}

\author{
Maria Voronkova $^{1 *}$, Evgeniy Banaev ${ }^{1}$, Maria Tomoshevich ${ }^{1}$ and Taigana AK-Lama ${ }^{1}$ \\ ${ }^{1}$ Central Siberian Botanical Garden of the Siberian Branch of the Russian Academy of Sciences, \\ 630090, Novosibirsk, Russian Federation
}

\begin{abstract}
The paper shows composition and content of phenolic compounds in leaves of 4 Nitraria L. species from 58 populations of Russia, Kazakhstan, and Tajikistan studied with high-performance liquid chromatography(HPLC). The investigation has revealed 27 compounds of phenolic nature: the maximum number (18 components) is detected in leaves of N. sibirica Pall. from Kazakhstan (the Karatal river valley), 16-17 components - in plants from three populations of Siberia, the minimum (6 components) - in leaves of $N$. komaroviilljin\& Lava ex Bobrov. The analysis has identified hyperoside (quercetin O-glycoside), narcissin (isoramnetin O-glycoside), quercetin (flavonol) and luteolin (flavon). The studied plants accumulate a generous quantity of phenolic compounds. Their content reaches $4.64 \%$ in leaves of $N$. sibirica, $3.11 \%$ - in $N$. schoberi L., up to $3.96 \%$ - in N. komarovii. The research results allow speaking about the species-specific composition and content of phenolic compounds of $N$. sibirica, $N$. schoberi and $N$. komarovii. The component composition is weaker in extracts of N. komarovii leaves, but there is a higher content of total phenolic compounds compared to N. schoberi plants. $N$. pamirica L. Vassil sample is close to $N$. schoberi on multidimensional analysis of the phenolic compound composition and content.
\end{abstract}

\section{Introduction}

The genus Nitraria L. (fam.Nitrariaceae) is represented by halophytic shrubs spread in steppe and desert areas of Minor and Central Asia, Southeast Europe, North Africa, and Australia. Species of this genus are promising medicinal plants. It is known antioxidant [1, 2], antigenotoxic [3], antiproliferative [4], cytotoxic [5, 6, 7, 8], antimicrobial, antiviral [9], antimutagenic, hypotensive [10], cardioprotective [11], hypolipidemic, hypoglycemic [1], hepatoprotective properties of these plant extracts [12] depending on a complex of biologically active substances in them. Secondary metabolites of the Nitraria L. genus plants are mainly represented by flavonoids and alkaloids, but the most researches are devoted to investigating alkaloids [12]. Along with studying useful properties, works are carried on to evaluate applying secondary metabolites of the Nitraria genus species as chemotaxonomic markers. Saleh et al. [13] have pointed to the relationship of alkaloid and

\footnotetext{
* Corresponding author: bmc_87@mail.ru
} 
flavonoid composition and the taxa systematic position at the family level. There is evidence that phenol-carboxylic acids and flavonoids in leaves of $N$. tangutorumBobr. are specific and can be used in the Nitraria genus taxonomy [5]. N. Barbhan et al. [8] have shown that the chemical composition of the aboveground part of $N$. retusa (Forsk.) Aschers. differs from other species of the Nitraria genus, which provides the base for further taxonomic and phylogenetic analysis. The work objective is to study the flavonoid composition and content in leaves of $N$. schoberi L., N. sibirica Pall., N. komaroviilljin\& Lava ex Bobrov, N.pamirica L. Vassil. in a wide geographical range, to interpret the obtained data in the chemotaxonomic aspect.

\section{Materials and Methods}

\subsection{Plant material}

The study material was collected in 58 Nirtariapopulations in Russia (Astrakhan region, Crimea, Siberia), Kazakhstan (Almaty, East Kazakhstan, Karaganda, Mangystau region) and Tajikistan (Eastern Pamir)in 2010-2017. Herbarium samples are stored in the Laboratory of Dendrology of the Central Siberian Botanical Garden of the Siberian Branch of the Russian Academy of Sciences (CSBG SB RAS).

\subsection{Isolation and identification of phenolic compounds}

Flavonoids were extracted by triple extraction with $70 \%$ ethanol when heated in a water bath. The analysis of individual phenolic compounds was carried on by HPLC analytical system consisting of Agilent 1200 chromatograph with a diode-matrix detector and ChemStation system. The technique detailed description is in papers [14, 15].Quantitative determination of individual glycosides and aglycones in eluates was performed using the external standard method in terms of quercetin $[14,15]$.

\section{Results and Discussion}

When studying the phenolic compound composition in plant leaves of N. schoberi, N. sibirica, N. komarovii, and N. pamirica, it has beenrevealed that the number of components in extractsvaries greatly. In general, 27 phenolic compounds mainly represented by flavonolglycosides are found in planleaves of thesespecies from various populations. By comparing the retention times of substance signals on chromatograms of analyzed samples with the retention times of standard samples and spectra, flavonol glycosides - hyperoside (quercetin-3-galactoside) and narcissin (isoramnetin-3-rutinoside), flavonol - quercetin, and flavon - luteolin are identified. The authors have noted compounds, which occurred in all studied species of Nitraria genus plants. The main components are: hyperoside, flavonol glycosides with retention times $t_{R}=3.1 ; t_{R}=15.8 ; t_{R}=34.1$; narcissin; quercetin and luteolin. Free quercetin is revealed in all studied Nitraria species, but it has not determined in 15 populations of $N$. sibirica and one population of $N$. komarovii. Quercetin is a precursor for its glycosides biosynthesis. Hyperoside, quercetin glycoside, is observed in all studied populations of all Nitraria species, except for one $N$. sibirica population from the Balkhash Lake region. Narcissin (isoramnetin-3-rutinoside) is marked in all Nitraria species under investigation, but it is poorly represented in spring leaves of $N$. sibirica (a small amount in only 3 populations). Despite the absence of some main components in plants of a number of $N$. sibirica populations, in general, this species is characterized by the richest compound composition in comparison with other studied species - up to 18 flavonoid components. 
N.sibiricaleaves are characterized by compounds with retention timesoft $\mathrm{R}_{\mathrm{R}}=6,4 ; \mathrm{t}_{\mathrm{R}}=8,7$; $t_{R}=9,3 ; t_{R}=10,2 ; t_{R}=11,3 ; t_{R}=27,8$, which are found in most populations of this species, while practically not identified in other species. Previously, we have already noted the species-specific composition of the phenolic compound complex for Siberian populations of $N$. schoberi and N. sibirica, which differences are especially evident in the flowering phase [14].

The phenolic composition of N. schoberi is weaker compared with N. sibirica. Plants of this species contain mainly no more than 14 compounds, the exception is the Saryozek population (16 compounds). The substance content of $N$. schoberi plants have lower concentrations as well. Leaves of $N$. sibiricasynthesize phenolic compounds (in terms of quercetin weight) from 1.30 to $4.64 \%$, depending on the growing place and development phase, an average total content of components is $3.02-3.45 \%$. The maximum quantity of components does not exceed 3\% in N. schoberi.

$N$. komarovii species has even weaker phenolic compound composition (no more than 7 components), but the content of the phenolic compounds sum during fruiting reaches $3.96 \%$. N. komarovii plants differ from other species by a significant content of flavonol glycosides with retention times of $\mathrm{t}_{\mathrm{R}}=3.1 ; \mathrm{t}_{\mathrm{R}}=4.7$ - up to $1.3 \%$ and $1.1 \%$, respectively. In $N$. sibirica, the flavonol glycoside content with a retention time of $\mathrm{tR}=3.1$ varies from $0.011 \%$ to $0.188 \%$ with an average population value of $0.1 \%$; with a retention time of $t_{R}=4.7$ - from $0.011 \%$ to $0.736 \%$ with an average population value of $0.3 \%$. N. schoberi leaves obtain substances with a retention time of $t_{R}=3.1$ from $0.029 \%$ to $0.739 \%$ with an average population value of $0.26 \%$; with a retention time of $t_{R}=4.7$ - from $0.153 \%$ to $0.522 \%$, with an average population value of $0.38 \%$.

N. pamirica species was collected in one population (East Pamir, Aktash Mountain Range). The dataon the phenolic compound composition and content in plant leaves of this population show their similarity to N. schoberi. 12 components are found in N. pamirica leaves, the amountof phenolic compounds is $2.44 \%$. Another 2 populations of Nitraria from the East Pamir (the Panj River valley) are also more similar to N. schoberi in the phenolic compound complex, while they are almost identical in the component composition and their content ( 9 phenolic components), the total content is 1.03 and $1.16 \%$.

Special attention should be paid to 8 Nitraria populations from Almaty Region of Kazakhstan, which are morphologically closer to $N$. sibirica, but differ from the latter species by the absence of phenolic components with retention time of $t_{R}=4.7$ and $t_{R}=6.5$. These components are identified in all 24 populations of $N$. sibirica from other regions. This fact, perhaps, should be regarded as the presence of ecological and geographical differentiation of $N$. sibirica populations.

The research has shown the presence of specificity in the phenolic compound composition and content of $N$. schoberi, N. sibirica, and N. komarovii. Thus, HPLC method can be used as a chemotaxonomic tool to solve issues of phylogeny and genesis of the Nitraria genus species. 


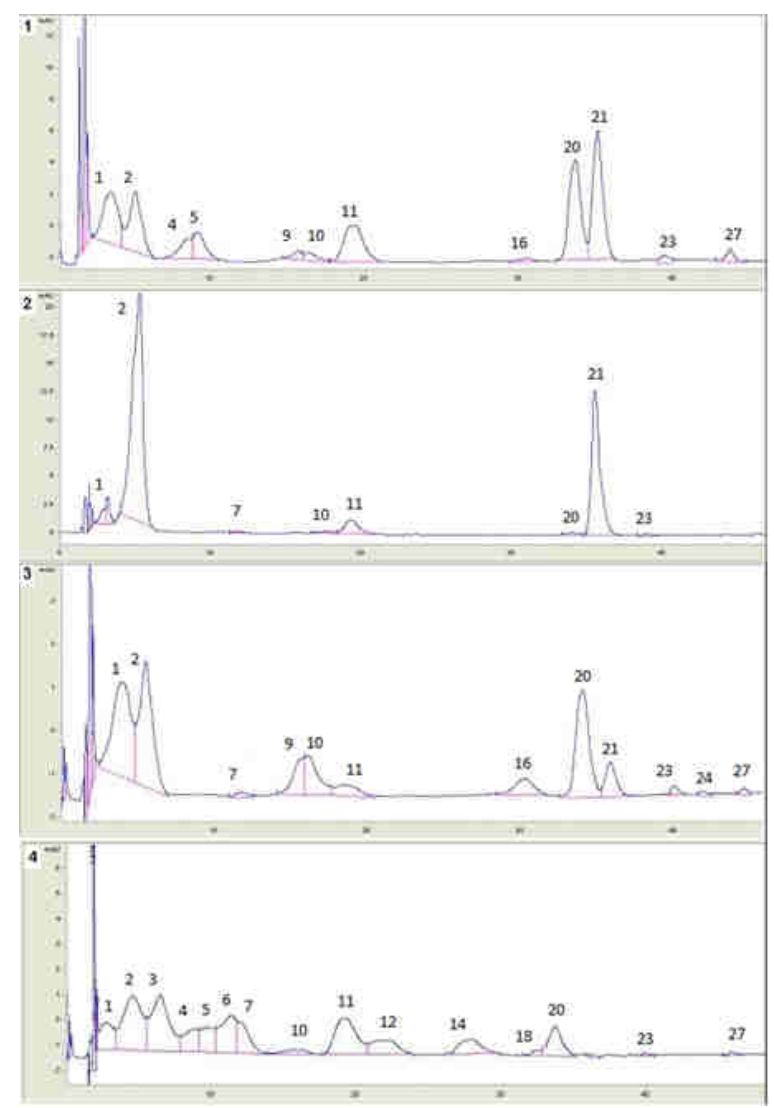

Fig. 1. Chromatograms of plant leaf extracts: $1-N$. schoberi (Balkhash Lake), $2-N$. komarovii (Balkhash Lake), $3-N$. pamirica (Aktash Range), $4-N$. sibirica (Jira population). The abscissus axis - retention time, $t_{R}$, min; the ordinate axis - optical density, o.d.u.

The research was carried out within the framework of the project VI.52.1.2 "Analysis of the intraspecific structure of resource plants in Asian Russia, the gene pool selection and conservation". State registration No AAAA-A17-117012610054-6.

\section{References}

1. J.-O. Zhao, Y.-M. Wang, Y.-L.Yang; Y. Zeng, Q.-L.Wang, Y. Shao, L.-J. Mei, Y.-P. Shi, Y.-D. Tao, Food Chem. 227, 93 - 101(2017)

2. G. Dongyu, Y. Yi, B. Mahinur, Q.-B. Chen, A.A. Haji, Natur. Product Res. 32, 1-4 (2017)

3. M.Chaabanea, M.Koubaac, N.Soudania, A.Elweja, M.Gratid, K.Jamoussid, T.Boudawarae, S.E. Chaabounib, N. Zeghala, Pharm. Biol. 55, 1061-1073 (2017)

4. J.Boubaker, I.M.Bzeouich, N.Nasr, H. B.Ghozlen, N.Mustapha,K.Ghedira, L.ChekirGhediraCompl.Altern. Med. 15, 300 (2015).

5. J.A. Duan, R.H. Zhou, S.X. Zhao J. Plant Resour Environ 8, 6-9 (1999)

6. T.Putkonen, A.Tolvanen, R.Jokela, S.Caccamese, N.Parrinello,Tetrahedron 59, 85898595 (2003) 
7. B.P.L.Liu, E.Y.Y.Chong, F.W.K.Cheung, J.A.Duan, C.T.Che, W.K.Liu,Biochem. Pharm. 70, 287-299(2005)

8. N.Barbhan, I.Miladi, S. I. Ali, E.Poupon, A. A. Mohamed, M. A. Beniddir. Chem.Natur. Compounds 53, 994-996 (2017)

9. T. Zheleznichenko, E. Banaev, S. Asbaganov, M. Voronkova, T.Kukushkina, E.Filippova, N. Mazurkova, L.Shishkina, T.Novikova. 3 Biotech 8, 260 (2018)

10. A.A.Ibragimov, Z.Osmanov, M.R.Iagudaev, S.Iu. IunusovKhim. Prirod. Soed. 2, $213-$ 216(1983) (in Russ.).

11. M. Zhang, J. Ma, H. Bi, J. Song, H. Yang, Z. Xia, Y. Du, T. Gao, L. Wei, Food a. Function 8, 2771 - 2782(2017)

12. Q.Du, H.Xin, Ch.Peng Mol. Med. Rep.11, 11-20(2015)

13. R.H.Sameh, A.K.Salwa, E.T.Mohamed, N.A.M. Saleh. Plant Syst. Evol. 277, 251264(2009)

14. E.V. Banaev, M.S. Voronkova, G.I. Vysochina, M.A. Tomoshevich, Contem. Probl. Ecol. 8, 735-742 (2015)

15. M.S. Voronkova, E.V. Banaev,M.A. Tomoshevich,Khim. Rast. Syriya 4, 107-116 (2017) 